\section{Contrast and the difference-of-logarithms}

\author{
THOMAS R. CORWIN \\ Center for Visual Science \\ University of Rochester \\ Rochester, New York 14627
}

In any visual scene composed of two or more simultaneous luminance values, we can identify regions of maximum $\left(\mathrm{L}_{\max }\right)$ and minimum $\left(\mathrm{L}_{\min }\right)$ luminance. From these two values, we can define the ratio $\mathbf{a}=\left(\mathrm{L}_{\max } / \mathrm{L}_{\min }\right)$, in terms of which we can express the scene's contrast $\mathrm{C}$ :

$C=\left(L_{\max }-L_{\min }\right) /\left(L_{\max }+L_{\min }\right)=(a-1) /(a+1)$.

Contrast is an important property because perceptual aspects of the scene seem to correlate more closely with $\mathrm{C}$ than with changes in $\mathrm{L}_{\max }$ or $\mathrm{L}_{\min }$ individually. In the phenomenon of contrast constancy, proportional changes in $\mathrm{L}_{\max }$ or $\mathrm{L}_{\min }$ (which do not alter the quantities a or C) leave the component regions of the scene unchanged in brightness, and so perceived contrast remains unchanged. Furthermore, when perceived contrast is measured as a function of physical contrast, the psychophysical relationship under many conditions is linear (Kulikowski, 1976). Contrast constancy can be accounted for by assuming that the visual system encodes contrast in terms of the quantity $\mathrm{C}$, or any single-valued function of $\mathrm{C}$, such as $\mathbf{a}$. The phenomenon of linearity of perceived contrast, however, constrains the hypothetical neural code for contrast with linear functions of $\mathrm{C}$. This rules out a as a possible code, since $\mathbf{a}$ is a highly nonlinear function of $\mathrm{C}$. How might the visual system produce a signal proportional to $C$ ? It does not seem physiologically parsimonious that the system would take Equation 1 literally, using separate neural networks to compute sums and differences and then to take their ratio. Is there a more plausible function of $L_{\max }$ and $\mathbf{L}_{\min }$ that is proportional or nearly proportional to C? Kulikowski (1976) has suggested the difference-of-logarithms function, in which contrast is encoded by subtracting the logarithms of $\mathrm{L}_{\max }$ and $\mathbf{L}_{\min }$. Since logarithmic transformations are known to occur at the receptor level, simple neural summation would be adequate to encode contrast. According to Kulikowski, the quantity $\left(\log \mathrm{L}_{\max }-\log \mathrm{L}_{\min }\right)$ $=\log \mathbf{a}$ is approximately proportional to the quantity $\mathrm{C}$, but he does not elaborate on the nature of the approximation. It is important to know the limits of this approximation, because they determine how well

Supported by NIH Grant EY02366 to T.R.C. and Core Center Grant EY01319 to the Center for Visual Science. a difference-of-logarithms code can account for the linear relationship between perceived and physical contrast.

The purpose of the present note is to determine how closely the difference-of-logarithms function approximates linearity with respect to $C$. This is conveniently done using the Taylor series expansion:

$$
\ln a=2\left[\frac{a-1}{a+1}+\frac{1}{3}\left(\frac{a-1}{a+1}\right)^{3}+\ldots\right] .
$$

Substitution from Equation 1 yields:

$$
\ln a=2\left(C+C^{3} / 3+C^{5} / 5+\ldots\right) .
$$

In this form, it can be seen that Kulikowski's hypothetical neural code is, in fact, closely proportional to $C$ when $C$ is small, because then the higher order terms of Equation 3 are negligible. In fact, these terms constitute no more than $10 \%$ of $C$ whenever $C$ is less than .50. However, as $\mathrm{C}$ approaches 1.0, the hypothetical neural code must increasingly depart from proportionality with $\mathrm{C}$, and in the limit $\mathrm{C}=1.0$, the difference-of-logarithms becomes infinite [since the harmonic series $(1+1 / 3+1 / 5+\ldots)$ diverges]. Despite its inability to encode contrasts close to 1.0 , the difference-of-logarithms code does account for the linear relationship between perceived and physical contrast for most ordinary contrasts.

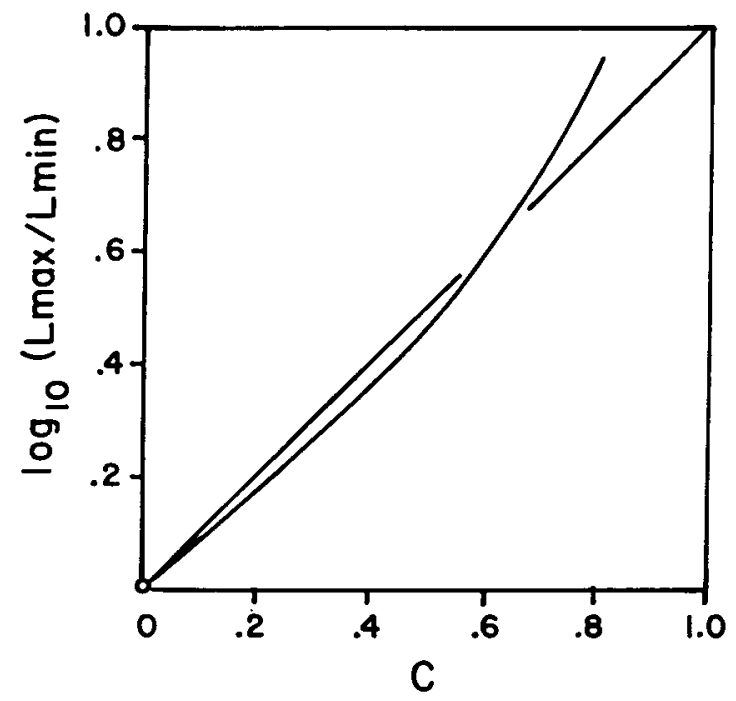

Figure 1. The difference-of-logarithms as a function of contrast when the logarithms are expressed to the base 10 . The diagonal line represents a strict identity between the difference-of-logarithms and contrast $(C)$. The difference-of-logarithms underestimates $C$ for all Cs less than approximately $\mathbf{. 6 0}$, and increasingly overestimates $\mathbf{C}$ as $\mathbf{C}$ approaches 1.0. The approximation is good to within $\pm 10 \%$ of $C$ for all values of $C$ between .32 and .73 , and to within $\pm 13.14 \%$ for all Cs less than $\mathbf{. 7 5 4}$. 
Although Equations 2 and 3 involve natural logarithms, the approximation to linearity with $C$ is not affected by a change in base, since this process requires only a proportionality constant. It is interesting, for example, to note that conversion to the common base-10 logarithm transforms the constant 2.0 in these equations to .867 , which is sufficiently close to 1.0 to provide a convenient way of estimating $\mathrm{C}$ by calculator. The approximation requires only two operations, whereas to calculate $\mathrm{C}$ exactly requires three different arithmetic operations and memory storage. The closeness of the relationship between $\log _{10} a$ and $C$ is illustrated graphically in Figure 1.
The relative ease with which the difference-oflogarithms can be calculated illustrates why the visual system might choose this parsimonious means to encode contrast.

\section{REFERENCES}

KULIKowski, J. J. Effective contrast constancy and linearity of contrast sensation. Vision Research, 1976, 16, 1419-1432.

(Received for publication September 10, 1980; accepted February 12, 1981.) 